\title{
Appling Genetic Algorithm to Optimize Locations and Activities of the Seeds in Brachytherapy to Deliver Dose to the Defined Border
}

\author{
Mowlavi AA ${ }^{1 *}$, Zibandeh-Gorji $\mathbf{M}^{2}$ and Mohammadi $\mathrm{S}^{2}$
}

${ }^{1}$ Physics Department, Sabsevar Tarbiat Moallem University, Sabzevar, Iran

${ }^{2}$ Physics Department, PhD center, Payame Noor University, Tehran, Iran

\begin{abstract}
As it is well known, improving in treatment planning of brachytherapy is very important. In this research, we have introduced a method for this purpose. We have applied the genetic algorithm to find the proper locations and activities of a ${ }^{125}$ brachytherapy seeds to deliver a desired dose to the border of the tumor in two dimensional surfaces. The initial positions of the seeds have been identified, and then by using a MATLAB code based on the genetic algorithm, we obtained the best locations and the activities of the seeds. This optimization method has been applied for some shape of the tumors. The results show that the desired dose to the border of a square, ellipsoid and general form of tumors is matched very well.
\end{abstract}

According to the results this technique can be used to optimize treatment plan of brachytherapy usefully. We plan to extend this method for three dimensional situations.

Keywords: ${ }^{125} \mathrm{I}$ seed; Dose distribution; Genetic algorithm; Optimization

\section{Introduction}

Although the first usage of ${ }^{125} \mathrm{I}$ isotopes was for diagnostic of thyroid disease; but this radioactive source has been used for diagnosis and treatment of many diseases especially in brachytherapy [1-6]. The isotopes used for permanent prostate implants are ${ }^{125} \mathrm{I}$ and ${ }^{103} \mathrm{Pd}$ which provide the tumor control. Permanent implantation of ${ }^{125} \mathrm{I}$ brachytherapy seeds has become an accepted and well-documented method for the treatment of prostate cancer. To have an adequate treatment factors such as activity of the seeds into the patient's body and seeds location within the tumor are very important [2-5]. These parameters can help us to understand the dose distribution around the sources accurately as well as are also very important in radiation therapy [6]. Recently, Singh et al. [7] have used an optimization algorithm to solve the brachytherapy seed localization problem in prostate brachytherapy.

\section{Material and Methods}

\section{Tumor shape}

In the first stage of this research, we have considered an image with 200 pixels $\times 200$ pixels $(5 \mathrm{~cm} \times 5 \mathrm{~cm})$ dimensions and set the spatial resolution $96 \times 96$ dots per inch square which is shown in Figure 1. This is ready to draw a tumor shape over this image square.

The aim of this research is finding proper locations of the seeds and their activity to deliver a desire dose to the general shape of tumor's border while all of the points inside the tumor receive the absorbed dose more than the dose value on the border.

\section{Radiation dose calculation}

In reality, iodine sources are cylindrical in shape with a physical length of $4.5 \mathrm{~mm}$ only with $0.8 \mathrm{~mm}$ diameter. The radioactive iodine is adsorbed on a silver rod $3 \mathrm{~mm}$ in length and encapsulated in a titanium capsule. But we used the point source approximation with different activity in our calculation. For this situation the dose depends the radial distance from the center of the source. The dose rate at a distance $r$ from a single point source is given by:

$$
\dot{D}(r)=\frac{S_{k} \Lambda}{r^{2}} g(r) \varphi_{a n}
$$

where $D(r)$ is the dose rate of the source in cGyh-1 unit, $\mathrm{S}_{\mathrm{k}}$ is the air kerma strength of the source measured in units of $U\left(1 U=1 \mu \mathrm{Gym}^{2} \mathrm{~h}^{-1}\right)$ as recommended by the AAPM Report No. 21 [8]. Also, the dose rate constant of the source is $\Lambda$ in $\mathrm{cGyh}^{-1} \mathrm{U}^{-1}$ unit, $\mathrm{r}$ is the radial distance of a point of interest from the source in centimeter unit, $g(r)$ is the radial dose function that accounts for the radial dependence of dose on the transverse axis due to photon absorption and scatter in the medium. Finally $\phi_{a n}$ is the anisotropy factor and is approximated by a constant in the point source dose formulation $[9,10]$. We have considered a ${ }^{125} \mathrm{I}$ source with $\Lambda=0.88 \mathrm{cGyh}^{-1} \mathrm{U}^{-1}, \phi_{\mathrm{an}}=0.0993$ parameters values which Meyer et al. [11] used in their used study. For purposes of simplicity, they have considered $S_{k}=1 \mathrm{U}$ and a fitted fifth-order polynomial of the form for the radial dose function of the source as:

$$
g(r)=a_{0}+a_{1} r+a_{2} r^{2}+a_{3} r^{3}+a_{4} r^{4}
$$

Where the coefficients are $a_{0}=1.01376 ; a_{1}=0.122747 ; a_{2}=$ $-0.173025 ; a_{3}=0.0402378 ; a_{4}=-0.00385227 ; a_{5}=0.000134283$.

The dose rate calculated using the point source approximation on the transverse axis (perpendicular to the cylindrical source axis) in the medium is lower than the actual close rate by $3-9 \%$ for most interstitial brachytherapy sources [11]. The total dose over time to tissue from a permanent radioactive implant is given by integrating the dose rate,

*Corresponding author: Mowlavi AA, Physics Department, Sabsevar Tarbiat Moallem University, Sabzevar, Iran, E-mail: aa_mowlavi@yahoo.com

Received November 04, 2011; Accepted December 16, 2011; Published December 18, 2011

Citation: Mowlavi AA, Zibandeh-Gorji M, Mohammadi S (2012) Appling Genetic Algorithm to Optimize Locations and Activities of the Seeds in Brachytherapy to Deliver Dose to the Defined Border. J Cancer Sci Ther 4: 001-003. doi:10.4172/1948-5956.1000100

Copyright: (c) 2012 Mowlavi AA, et al. This is an open-access article distributed under the terms of the Creative Commons Attribution License, which permits unrestricted use, distribution, and reproduction in any medium, provided the original author and source are credited. 
Citation: Mowlavi AA, Zibandeh-Gorji M, Mohammadi S (2012) Appling Genetic Algorithm to Optimize Locations and Activities of the Seeds in Brachytherapy to Deliver Dose to the Defined Border. J Cancer Sci Ther 4: 001-003. doi:10.4172/1948-5956.1000100

taking into account the half-life $\left(\mathrm{T}_{1 / 2}\right)$ of the radioactive source. The total dose from a permanently implanted source is [8]:

$$
D(r)=\int_{0}^{\infty} \dot{D}(r) \exp \left(\frac{-0.693 t}{T_{1 / 2}}\right) d t \Rightarrow D(r) \cong 1.44 T_{1_{1 / 2}} \dot{D}(r)
$$

The total dose $\mathrm{D}_{\mathrm{ij}}$ delivered by all seeds to the pixel (i $\left.\mathrm{j}\right)$, in the grid, is given by:

$$
D_{i j}=\sum_{k=1}^{S_{n}} w_{i} d_{i j}^{p}
$$

Where $\mathrm{d}^{\mathrm{p}}{ }_{\mathrm{ij}}$ is the dose contribution from the seed at grid location $\mathrm{P}$, and $S_{n}$ is the total number of the seeds. In Figure 1 three seeds are presented in the pixel locations, and it is well known that the dose to the pixel $(i, j)$ in the two-dimensional matrix is the summation of the doses from individual seeds 1,2 , and 3 to pixel $(i, j)$.

\section{Genetic Algorithms}

When using a genetic algorithm with a vector of $3 S_{n}$ variables that take on us the first $S_{n}$ variables related to the x coordinate of the seeds location and $S_{n}$ variables related to the y coordinate of the seeds location and the last $S_{n}$ variables are the weights which related to the activities of the seeds as the weight values. In this case, each chromosome is made of three parts that each section has nine genes which is the general form is as follows:

$$
c=\left(x_{1}+x_{2}, \ldots, x_{S n}+y_{1}+y_{2}, \ldots, y_{S n}+w_{1}+w_{2}, \ldots, w_{S n}\right)
$$

With proper selection and implementation parameters required the genetic algorithm code, it can possible to do the optimization

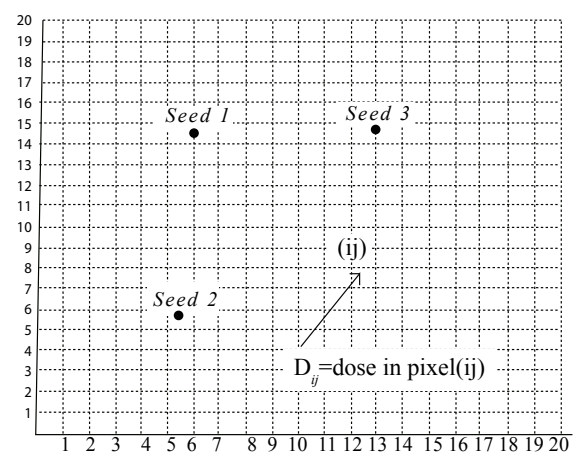

Figure 1: Total radiation dose to each point $(\mathrm{ij})$ in the grid is obtained by summing contributions from the all seeds.

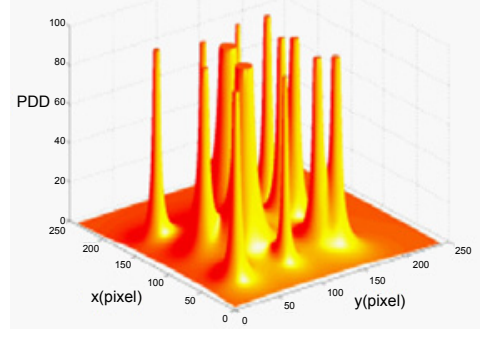

(a)

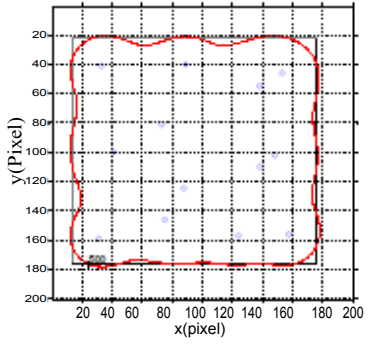

(b)
Figure 2: a) The three dimensional PDD variation from 13 seeds with different activity; b) the obtained dose curve is on many parts of the body matches the square border. calculation and find the proper seeds locations and their activities. The initial positions of the seeds have been identified very simply like over the grid points, and then by using the genetic algorithm code that we wrote in MATLAB, the best locations and activities of the seeds to reach the border dose as a wanted value are found.

\section{Results and Discussions}

As it mentioned before, we have applied the genetic algorithm code to find the proper locations and activities of ${ }^{125}$ I seeds in brachytherapy to deliver a desired dose to the border of the tumor in two dimensional surfaces. Figure 2 shows the results of the optimization for reaching a desire doe to a square border. The three dimensional percentage deep dose (PDD) variation is shown in Figure $2 \mathrm{a}$ and the match of desire dose curve with the square border is presented in Figure $2 \mathrm{~b}$. As well as, the obtained result including the location coordinates of 13 seeds and the amount of their weight values after running of the genetic algorithms are listed in Table 1. The same results for ellipsoid border have been

\begin{tabular}{|c|c|c|}
\hline$X$ & $Y$ & $w$ \\
\hline 146.00 & 75.00 & 0.03 \\
156.00 & 157.42 & 0.05 \\
40.00 & 89.00 & 0.02 \\
55.00 & 138.00 & 0.06 \\
157.00 & 124.00 & 0.02 \\
110.01 & 138.23 & 0.04 \\
46.00 & 153.00 & 0.06 \\
41.00 & 32.73 & 0.05 \\
100.00 & 41.00 & 0.06 \\
159.00 & 31.00 & 0.04 \\
102.00 & 148.00 & 0.06 \\
81.00 & 73.00 & 0.26 \\
124.60 & 87.56 & 0.25 \\
\hline
\end{tabular}

Table 1: The location coordinates of each seed and the amount of the weight values after running of the genetic algorithm.
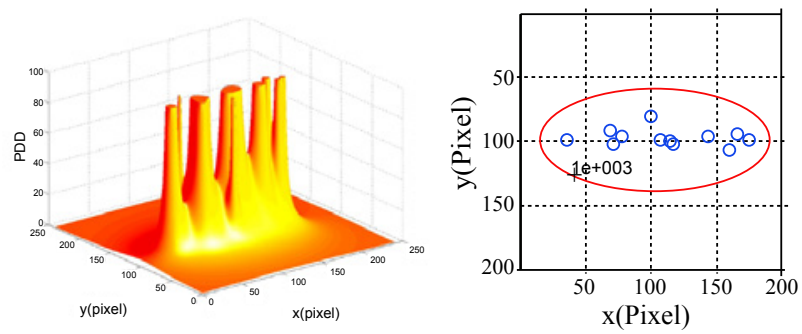

(a)

Figure 3: a) Three dimensional PDD variation in $\mathrm{z}=0 \mathrm{~mm}$ surface, $\mathrm{b}$ ) the obtained dose curve is very well to the body matches the ellipsoid border.

\begin{tabular}{|c|c|c|}
\hline $\mathrm{x}$ & $\mathrm{Y}$ & $\mathrm{W}$ \\
\hline 91.00 & 68.47 & 0.07 \\
97.00 & 78.16 & 0.04 \\
117.00 & 61.00 & 0.01 \\
102.00 & 71.68 & 0.15 \\
99.96 & 113.53 & 0.12 \\
106.00 & 160.00 & 0.05 \\
98.34 & 175.00 & 0.03 \\
99.00 & 37.00 & 0.12 \\
80.00 & 99.78 & 0.01 \\
102.00 & 116.00 & 0.15 \\
95.00 & 167.00 & 0.03 \\
96.00 & 144.00 & 0.14 \\
99.00 & 106.00 & 0.07 \\
\hline
\end{tabular}

Table 2: The location coordinates of 13 seeds and the amount of the weight values after running the genetic algorithm. 
Citation: Mowlavi AA, Zibandeh-Gorji M, Mohammadi S (2012) Appling Genetic Algorithm to Optimize Locations and Activities of the Seeds in Brachytherapy to Deliver Dose to the Defined Border. J Cancer Sci Ther 4: 001-003. doi:10.4172/1948-5956.1000100

\begin{tabular}{|c|c|c|}
\hline$X$ & $Y$ & $w$ \\
\hline 45.00 & 114.00 & 0.03 \\
155.00 & 40.00 & 0.06 \\
169.00 & 48.00 & 0.03 \\
41.00 & 112.00 & 0.02 \\
113.00 & 86.00 & 0.02 \\
182.00 & 64.00 & 0.03 \\
126.00 & 56.00 & 0.03 \\
95.00 & 120.00 & 0.05 \\
42.00 & 108.00 & 0.04 \\
154.00 & 40.00 & 0.03 \\
59.00 & 123.00 & 0.18 \\
157.00 & 39.00 & 0.04 \\
126.00 & 55.00 & 0.02 \\
127.00 & 56.00 & 0.03 \\
164.00 & 44.00 & 0.04 \\
156.00 & 41.00 & 0.03 \\
150.00 & 40.49 & 0.08 \\
59.00 & 120.00 & 0.08 \\
65.00 & 123.00 & 0.18 \\
\hline
\end{tabular}

2. Udale-Smith M (1992) Monte Carlo calculations of electron beam parameters for three Philips linear accelerators. Phys Med Biol 37: 85-105.

3. Karaiskos $\mathrm{P}$, Angelopoulos A, Baras $\mathrm{P}$, Rozaki-Mavrouli $\mathrm{H}$, Sandilos $\mathrm{P}$, et al. (2000) Dose rate calculations around ${ }^{192}$ Ir brachytherapy sources using a Sievert integration model. Phys Med Biol 45: 383-398.

4. Gifford KA, Mourtada F, Cho SH, Lawyer A, Horton JL (2005) Monte Carlo calculations of the dose distribution around a commercial gynecologic tandem applicator. Radiother Oncol 77: 210-215.

5. Mowlavi AA, Cupardo F, Severgnini M (2008) Monte Carlo and experimental relative dose determination for an Ir-192 source in water phantom. Iran J Radiat Res 6: 37-42.

6. Ling CC, Schell MC, Yorke ED, Palos BB, Kubiatowicz DO (1985) Twodimensional dose distribution of ${ }^{125} \mathrm{I}$ seeds. Med Phys 12: 652-655.

7. Singh V, Mukherjee L, Xu J, Hoffmann KR, Dinu PM, et al. (2007) Brachytherapy seed localization using geometric and linear programming techniques. IEEE Trans Med Imaging 26: 1291-1304.

Table 3: The location coordinates of 19 seeds and the amount of the weight value for each seed after applying genetic algorithm.

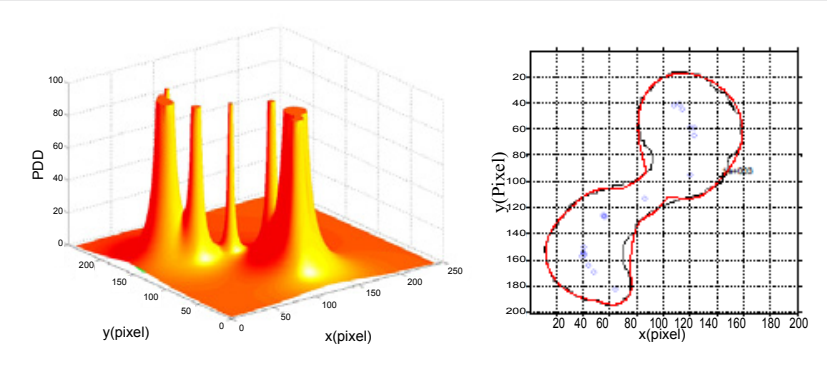

Figure 4: a) Three dimensional PDD variation in $\mathrm{z}=0 \mathrm{~mm}$ surface, $\mathrm{b}$ ) the obtained desire dose curve is matched very well to the general complex border.

presented in Figure 3 which the seeds locations with corresponding weights are listed in Table 2,3. It can be seen that the obtained results in this sample are very well mashed with the ellipsoid border.

We have also applied the technique for a general form of tumor border as shown in Figure 4 . It can be seen that the result are well covered the border, just in some part a little difference is appear.

The results show that the genetic algorithm code can be applied for any tumor border shape accurately.

\section{Conclusion}

We have applied the genetic algorithm code to find the proper positions and activities of ${ }^{125} \mathrm{I}$ brachytherapy seeds to deliver a desired dose to the border of the tumor in two dimensional surfaces while all of the points inside the tumor receive the absorbed dose more than the dose value on the border. According to the obtained results, the technique can be used tor optimize treatment plan of brachytherapy usefully. The results show that it is possible to apply genetic algorithm technique for any geometric shape in two-dimensional space. Finally, the results in two dimension surface are interesting, and guide us to extend this method to real shape of tumors in three dimensional situations.

\section{References}

1. Hafeli UO, Roberts WK, Meier DS, Ciezki J, Pauer GJ, et al. (2000) Dosimetry of a W-188/Re-188 beta line source for endovascular brachytherapy. Med Phys 27: 668-675.

8. AAPM Report No. 21, Recommendations of AAPM Task Group 32: Specification of Brachytherapy Source Strength. American Institute of Physics, New York, 1987.

9. Williamson JF, Thomadsen BR, Nath R (1995) Brachytherapy Physics. Medical Physics Publishing, Madison WI 7-37.

10. Nath R, Anderson LL, Luxton G, Weaver KA, Williamson JF, et al. (1995) Dosimetry of interstitial brachytherapy sources: recommendations of the AAPM Radiation Therapy Committee Task Group No. 43. American Association of Physicists in Medicine. Med Phys 22: 209-234.

11. Meyer RR, D'Souza WD, Ferris MC, Thomadsen BR (2003) MIP models and BB strategies in brachytherapy treatment optimization. J Glob optim 25: 23-42. 